\title{
The constructions of general connections on the fibred product of $q$ copies of the first jet prolongation
}

\begin{abstract}
We describe all natural operators $A$ transforming general connections $\Gamma$ on fibred manifolds $Y \rightarrow M$ and torsion-free classical linear connections $\Lambda$ on $M$ into general connections $A(\Gamma, \Lambda)$ on the fibred product $J^{<q>} Y \rightarrow M$ of $q$ copies of the first jet prolongation $J^{1} Y \rightarrow M$.
\end{abstract}

1. Introduction. All manifolds are smooth, Hausdorff, finite dimensional and without boundaries. Maps are assumed to be smooth, i.e. of class $C^{\infty}$.

The concept of $r$-th order connections for arbitrary fibred manifolds was introduced by I. Kolář in [3].

Let us recall that an $r$-th order connection on a fibred manifold $p: Y \rightarrow$ $M$ is a section $\Theta: Y \rightarrow J^{r} Y$ of the $r$-jet prolongation $\beta: J^{r} Y \rightarrow Y$ of $p: Y \rightarrow M$. A general connection on $p: Y \rightarrow M$ is a first order connection $\Gamma: Y \rightarrow J^{1} Y$ or (equivalently) a lifting map

$$
\Gamma: Y \times_{M} T M \rightarrow T Y .
$$

By $\operatorname{Con}(Y \rightarrow M)$ we denote the set of all general connections on a fibred manifold $p: Y \rightarrow M$.

2010 Mathematics Subject Classification. 58A05, 58A20, 58A32.

Key words and phrases. General connection, classical linear connection, first jet prolongation, bundle functor, natural operator. 
If $p: Y \rightarrow M$ is a vector bundle and an $r$-th order connection $\Theta: Y \rightarrow$ $J^{r} Y$ is a vector bundle morphism, then $\Theta$ is called an $r$-th order linear connection on $p: Y \rightarrow M$.

An $r$-th order linear connection on $M$ is an $r$-th order linear connection $\Lambda: T M \rightarrow J^{r} T M$ on the tangent bundle $\pi_{M}: T M \rightarrow M$ of $M$. By $Q^{r}(M)$ we denote the set of all $r$-th order linear connections on $M$.

A classical linear connection on $M$ is a first order linear connection $\nabla: T M \rightarrow J^{1} T M$ on $M$, which can be also (equivalently) considered as its corresponding covariant derivative $\nabla: \mathfrak{X}(M) \times \mathfrak{X}(M) \rightarrow \mathfrak{X}(M)$.

A classical linear connection $\nabla$ on $M$ is called torsion-free if its torsion tensor $T(X, Y)=\nabla_{X} Y-\nabla_{Y} X-[X, Y]$ is equal to zero. By $Q_{\tau}(M)$ we denote the set of all torsion-free classical linear connections on $M$.

Let $\mathcal{F M}$ denote the category of fibred manifolds and their fibred maps and let $\mathcal{F M}_{m, n} \subset \mathcal{F} \mathcal{M}$ be the (sub)category of fibred manifolds with $m$ dimensional bases and $n$-dimensional fibres and their local fibred diffeomorphisms. Let $\mathcal{M} f_{m}$ denote the category of $m$-dimensional manifolds and their local diffeomorphisms.

Let $F: \mathcal{F} \mathcal{M}_{m, n} \rightarrow \mathcal{F M}$ be a bundle functor on $\mathcal{F} \mathcal{M}_{m, n}$ of order $r$ in the sense of [4]. Let $\Gamma: Y \times_{M} T M \rightarrow T Y$ be the lifting map of a general connection on an $\mathcal{F} \mathcal{M}_{m, n}$-object $p: Y \rightarrow M$. Let $\Lambda: T M \rightarrow J^{r} T M$ be an $r$-th order linear connection on $M$. The flow operator $\mathcal{F}$ of $F$ transforming projectable vector fields $\eta$ on $p: Y \rightarrow M$ into vector fields $\mathcal{F} \eta:=\frac{\partial}{\partial t} \mid t=0, F\left(F l_{t}^{\eta}\right)$ on $F Y$ is of order $r$. In other words, the value $\mathcal{F} \eta(u)$ at every $u \in F_{y} Y, y \in Y$ depends only on $j_{y}^{r} \eta$. Therefore, we have the corresponding flow morphism $\tilde{\mathcal{F}}: F Y \times_{Y} J^{r} T Y \rightarrow T F Y$, which is linear with respect to $J^{r} T Y$. Moreover, $\tilde{\mathcal{F}}\left(u, j_{y}^{r} \eta\right)=\mathcal{F} \eta(u)$, where $u \in F_{y} Y, y \in Y$. Let $X^{\Gamma}$ be the $\Gamma$-lift of a vector field $X$ on $M$ to $Y$, i.e. $X^{\Gamma}$ is a projectable vector field on $p: Y \rightarrow M$ defined by $X^{\Gamma}(y)=\Gamma(y, X(x)), y \in Y_{x}$, $x=p(y) \in M$. Then the connection $\Gamma$ can be extended to a morphism $\tilde{\Gamma}: Y \times_{M} J^{r} T M \rightarrow J^{r} T Y$ by the following formula $\tilde{\Gamma}\left(y, j_{x}^{r} X\right)=j_{y}^{r}\left(X^{\Gamma}\right)$. By applying $\mathcal{F}$, we obtain a map $\mathcal{F}(\tilde{\Gamma}): F Y \times_{M} J^{r} T M \rightarrow T F Y$ defined by $\mathcal{F}(\tilde{\Gamma})\left(u, j_{x}^{r} X\right)=\tilde{\mathcal{F}}\left(u, j_{y}^{r}\left(X^{\Gamma}\right)\right)=\mathcal{F} X^{\Gamma}(u)$. Further, the composition

$$
\mathcal{F}(\Gamma, \Lambda):=\mathcal{F}(\tilde{\Gamma}) \circ\left(i d_{F Y} \times \Lambda\right): F Y \times_{M} T M \rightarrow T F Y
$$

is the lifting map of a general connection on $F Y \rightarrow M$. The connection $\mathcal{F}(\Gamma, \Lambda)$ is called $F$-prolongation of $\Gamma$ with respect to $\Lambda$ and was discovered by I. Kolář [2].

In particular, if $F: \mathcal{F} \mathcal{M}_{m, n} \rightarrow \mathcal{F M}$ is a bundle functor on $\mathcal{F} \mathcal{M}_{m, n}$ of order $r=1$ and $\Gamma$ is a general connection on an $\mathcal{F} \mathcal{M}_{m, n}$-object $p: Y \rightarrow M$ and $\nabla$ is a torsion-free classical linear connection on $M$, then one can obtain the general connection $\mathcal{F}(\Gamma, \nabla)$ as in $[2]$. 
In the paper [1] authors introduced some interesting constructions on connections using other methods.

2. Natural operators. The canonical character of construction of this connection can be described by means of the concept of natural operators. The general concept of natural operators can be found in the fundamental monograph [4]. In particular, we have the following definitions.

Definition 1. Let $F: \mathcal{F} \mathcal{M}_{m, n} \rightarrow \mathcal{F M}$ be a bundle functor of order $r=1$ on the category $\mathcal{F} \mathcal{M}_{m, n}$ and $\mathcal{B}: \mathcal{F} \mathcal{M}_{m, n} \rightarrow \mathcal{M} f_{m}$ be a base functor. An $\mathcal{F} \mathcal{M}_{m, n}$-natural operator $D: J^{1} \times Q_{\tau}(\mathcal{B}) \rightsquigarrow J^{1}(F \rightarrow \mathcal{B})$ transforming general connections $\Gamma$ on fibred manifolds $Y \rightarrow M$ and torsion-free classical linear connections $\nabla$ on $M$ into general connections $D(\Gamma, \nabla): F Y \rightarrow J^{1} F Y$ on $F Y \rightarrow M$ is a system of regular operators $D_{Y}$ : $\operatorname{Con}(Y \rightarrow M) \times Q_{\tau}(M) \rightarrow$ $\operatorname{Con}(F Y \rightarrow M),(p: Y \rightarrow M) \in \operatorname{Obj}\left(\mathcal{F} \mathcal{M}_{m, n}\right)$ satisfying the $\mathcal{F} \mathcal{M}_{m, n^{-}}$ invariance condition.

The $\mathcal{F} \mathcal{M}_{m, n}$-invariance means that for any connections $\Gamma \in \operatorname{Con}(Y \rightarrow$ $M), \Gamma_{1} \in \operatorname{Con}\left(Y_{1} \rightarrow M_{1}\right), \nabla \in Q_{\tau}(M)$ and $\nabla_{1} \in Q_{\tau}\left(M_{1}\right)$ such that if $\Gamma$ is $f$-related to $\Gamma_{1}$ by an $\mathcal{F} \mathcal{M}_{m, n}$-map $f: Y \rightarrow Y_{1}$ covering $f: M \rightarrow$ $M_{1}$ (i.e. $J^{1} f \circ \Gamma=\Gamma_{1} \circ f$ ) and $\nabla$ is $\underline{f}$-related to $\nabla_{1}$ (i.e. $J^{1} T \underline{f} \circ \nabla=$ $\nabla_{1} \circ T f$ ), then $D_{Y}(\Gamma, \nabla)$ is $F f$-related to $D_{Y_{1}}\left(\Gamma_{1}, \nabla_{1}\right)$ (i.e. $J^{1} F f \circ \bar{D}_{Y}(\Gamma, \nabla)$ $\left.=D_{Y_{1}}\left(\Gamma_{1}, \nabla_{1}\right) \circ F f\right)$.

Equivalently the $\mathcal{F} \mathcal{M}_{m, n}$-invariance means that for any $\Gamma \in \operatorname{Con}(Y \rightarrow$ $M), \Gamma_{1} \in \operatorname{Con}\left(Y_{1} \rightarrow M_{1}\right), \nabla \in Q_{\tau}(M)$ and $\nabla_{1} \in Q_{\tau}\left(M_{1}\right)$ if diagrams
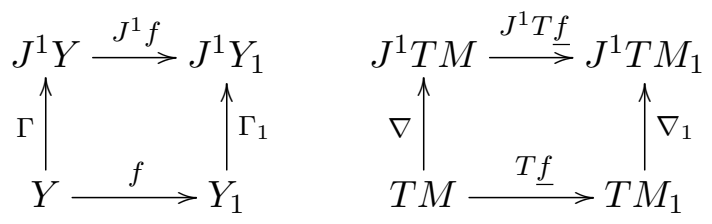

commute for an $\mathcal{F M}_{m, n}$-map $f: Y \rightarrow Y_{1}$ covering $\underline{f}: M \rightarrow M_{1}$, then the diagram

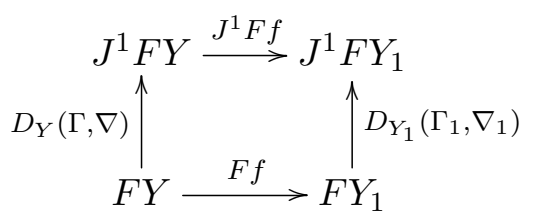

commutes.

We say that the operator $D_{Y}$ is regular if it transforms smoothly parametrized families of connections into smoothly parametrized ones.

Thus the construction $\mathcal{F}(\Gamma, \nabla)$ can be considered as an $\mathcal{F} \mathcal{M}_{m, n}$-natural operator $\mathcal{F}: J^{1} \times Q_{\tau}(\mathcal{B}) \rightsquigarrow J^{1}(F \rightarrow \mathcal{B})$. 
3. Quasi-normal fibred coordinates. According to [6], let

$$
\Phi_{r}: J_{0}^{r-1}\left(T^{*} \mathbb{R}^{m} \otimes \mathbb{R}^{n}\right) \rightarrow J_{0}^{r}\left(\mathbb{R}^{m}, \mathbb{R}^{n}\right)_{0}
$$

be the usual symmetrization

$$
\bigoplus_{q=0}^{r-1} S^{q} T_{0}^{*} \mathbb{R}^{m} \otimes T_{0}^{*} \mathbb{R}^{m} \otimes \mathbb{R}^{n} \rightarrow \bigoplus_{q=0}^{r-1} S^{q+1} T_{0}^{*} \mathbb{R}^{m} \otimes \mathbb{R}^{n}
$$

modulo the following $G L(m)$-invariant identifications:

$$
\begin{aligned}
& J_{0}^{r-1}\left(T^{*} \mathbb{R}^{m} \otimes \mathbb{R}^{n}\right)=\bigoplus_{q=0}^{r-1} S^{q} T_{0}^{*} \mathbb{R}^{m} \otimes T_{0}^{*} \mathbb{R}^{m} \otimes \mathbb{R}^{n}, \\
& J_{0}^{r}\left(\mathbb{R}^{m}, \mathbb{R}^{n}\right)_{0}=\bigoplus_{q=0}^{r-1} S^{q+1} T_{0}^{*} \mathbb{R}^{m} \otimes \mathbb{R}^{n} .
\end{aligned}
$$

In other words, $\Phi_{r}: J_{0}^{r-1}\left(T^{*} \mathbb{R}^{m} \otimes \mathbb{R}^{n}\right) \rightarrow J_{0}^{r}\left(\mathbb{R}^{m}, \mathbb{R}^{n}\right)_{0}$ is the linear map such that

$$
\Phi_{r}\left(j_{0}^{r-1}\left(\left(x^{i_{1}} \ldots x^{i_{q}} d x^{j}\right) e_{k}\right)\right)=\frac{1}{q+1} j_{0}^{r}\left(x^{i_{1}} \ldots x^{i_{q}} x^{j} e_{k}\right)
$$

for any $i_{1}, \ldots, i_{q}, j=1, \ldots, m, q=0, \ldots, r-1$ and $k=1, \ldots, n$, where $\left(e_{k}\right)$ is the usual canonical basis in $\mathbb{R}^{n}$ and $\left(x^{1}, \ldots, x^{m}\right)$ are the usual coordinates on $\mathbb{R}^{m}$. Then it holds

$$
\Phi_{r}\left(j_{0}^{r-1}(d \sigma)\right)=j_{0}^{r}(\sigma)
$$

for any $\sigma: \mathbb{R}^{m} \rightarrow \mathbb{R}^{n}$ with $\sigma(0)=0$. In addition, $\Phi_{r}$ is $G L(m)$-invariant and linear.

Let $\Gamma: Y \rightarrow J^{1} Y$ be a general connection on a fibred manifold $p: Y \rightarrow M$, where $\operatorname{dim}(M)=m, \operatorname{dim}(Y)=m+n$. Let $\Lambda$ be a torsion-free classical linear connection on $M$. Let $y_{0} \in Y$ be a point such that $x_{0}=p\left(y_{0}\right) \in M$.

We present a concept of $\left(\Gamma, \Lambda, y_{0}, r\right)$-quasi-normal fibred coordinate system on $Y$, which was introduced by W. Mikulski, [6], [7].

Definition 2. A fibred chart $\psi$ on $Y$ with $\psi\left(y_{0}\right)=(0,0) \in \mathbb{R}^{m, n}$ covering a $\Lambda$-normal coordinate system $\psi$ on $M$ with centre $x_{0}$ is called a $\left(\Gamma, \Lambda, y_{0}, r\right)$ quasi-normal fibred coordinate system on $Y$, if the condition

$$
\Phi_{r}\left(j_{0}^{r-1}\left(\sum_{|\alpha|+|\beta| \leq r-1} \sum_{j=1}^{m} \sum_{k=1}^{n} \Gamma_{j \alpha \beta}^{k} x^{\alpha} d x^{j} \otimes e_{k}\right)\right)=0
$$

holds for any multiindex $\beta \in(\mathbb{N} \cup\{0\})^{n}$ such that $|\beta| \leq r-1$, where

$$
j_{0}^{r-1}\left(\sum_{i=1}^{m} d x^{i} \otimes \frac{\partial}{\partial x^{i}}+\sum_{|\alpha|+|\beta| \leq r-1} \sum_{j=1}^{m} \sum_{k=1}^{n} \Gamma_{j \alpha \beta}^{k} x^{\alpha} y^{\beta} d x^{j} \otimes \frac{\partial}{\partial y^{k}}\right)
$$


is the expression of an element $j_{(0,0)}^{r-1}\left(\psi_{*} \Gamma\right)$ and $\left(x^{1}, \ldots, x^{m}, y^{1}, \ldots, y^{n}\right)$ are the usual coordinates on the product $\mathbb{R}^{m} \times \mathbb{R}^{n}$.

In [6], W. Mikulski proved the following theorem.

Theorem 1. Let $\Gamma: Y \rightarrow J^{1} Y$ be a general connection on an $\mathcal{F M}_{m, n^{-}}$ object $p: Y \rightarrow M$ such that $\operatorname{dim}(M)=m, \operatorname{dim}(Y)=m+n$ and let $\Lambda$ be a torsion-free classical linear connection on $M$ and let $y_{0} \in Y$ be a point such that $x_{0}=p\left(y_{0}\right) \in M$. Then:

(i) There exists a $\left(\Gamma, \Lambda, y_{0}, r\right)$-quasi-normal fibred coordinate system $\psi$ on $Y$. (ii) If $\psi^{1}$ is another $\left(\Gamma, \Lambda, y_{0}, r\right)$-quasi-normal fibred coordinate system on $Y$, then

$$
j_{y_{0}}^{r} \psi^{1}=j_{y_{0}}^{r}((B \times H) \circ \psi)
$$

for a map $B \in G L(m)$ and a diffeomorphism $H: \mathbb{R}^{n} \rightarrow \mathbb{R}^{n}$ preserving $0 \in \mathbb{R}^{n}$.

From the proof of this theorem it follows that $(B \times H) \circ \psi$ is a $\left(\Gamma, \Lambda, y_{0}, r\right)$ quasi-normal fibred coordinate system on $Y$ for any $B \in G L(m)$ and any diffeomorphism $H: \mathbb{R}^{n} \rightarrow \mathbb{R}^{n}$ preserving $0 \in \mathbb{R}^{n}$. In other words, the $\mathcal{F} \mathcal{M}_{m, n}$-maps of the form $B \times H$ for $B \in G L(m)$ and diffeomorphisms $H: \mathbb{R}^{n} \rightarrow \mathbb{R}^{n}$ preserving $0 \in \mathbb{R}^{n}$ transform $\left(\Gamma, \Lambda, y_{0}, r\right)$-quasi-normal fibred coordinate systems on $Y$ into $\left(\Gamma, \Lambda, y_{0}, r\right)$-quasi-normal fibred coordinate systems.

The generalization of this theorem in the case $r=2$ for fibred-fibred manifolds was proved by J. Kurek and W. Mikulski in [5].

4. The fibred product of $q$ copies of the first jet prolongation. In [4], the authors described all $\mathcal{F} \mathcal{M}_{m, n}$-natural operators $D: J^{1} \times Q_{\tau}(\mathcal{B})$ $\rightsquigarrow J^{1}(F \rightarrow \mathcal{B})$ for a bundle functor $F=J^{1}: \mathcal{F} \mathcal{M}_{m, n} \rightarrow \mathcal{F} \mathcal{M}$. They constructed an additional $\mathcal{F} \mathcal{M}_{m, n}$-natural operator $P$ and proved that all $\mathcal{F} \mathcal{M}_{m, n}$-natural operators $D: J^{1} \times Q_{\tau}(\mathcal{B}) \rightsquigarrow J^{1}\left(J^{1} \rightarrow \mathcal{B}\right)$ form the one parameter family $t P+(1-t) \mathcal{J}^{1}, t \in \mathbb{R}$.

In other words, they showed that any $\mathcal{F} \mathcal{M}_{m, n}$-natural operator

$$
C: J^{1} \times Q_{\tau}(\mathcal{B}) \rightsquigarrow J^{1}\left(J^{1} \rightarrow \mathcal{B}\right)
$$

transforming pairs $(\Gamma, \Lambda)$ consisting of general connections $\Gamma: Y \rightarrow J^{1} Y$ on $\mathcal{F} \mathcal{M}_{m, n}$-objects $p: Y \rightarrow M$ and torsion-free classical linear connections $\Lambda: T M \rightarrow J^{1} T M$ on $M$ into general connections $C_{Y}(\Gamma, \Lambda): J^{1} Y \rightarrow J^{1} J^{1} Y$ on $J^{1} Y \rightarrow M$ is of the form

$$
C=t \cdot P+(1-t) \cdot \mathcal{J}^{1}, t \in \mathbb{R},
$$

where $P$ and $\mathcal{J}^{1}$ are natural operators constructed in the monograph [4].

In [8], we generalized this result to the case $F=J^{2}$. In other words, we classified all $\mathcal{F} \mathcal{M}_{m, n}$-natural operators $D: J^{1} \times Q_{\tau}(\mathcal{B}) \rightsquigarrow J^{1}\left(J^{2} \rightarrow \mathcal{B}\right)$. 
A pair $(\Gamma, \Lambda)$ consisting of a general connection $\Gamma: Y \rightarrow J^{1} Y$ on a fibred manifold $p: Y \rightarrow M$ and a torsion-free classical linear connection $\Lambda: T M \rightarrow$ $J^{1} T M$ on $M$ is called an admissible pair on $p: Y \rightarrow M$.

We can consider the first jet prolongation functor $J^{1}$ as an affine bundle functor on the category $\mathcal{F} \mathcal{M}_{m, n}$. The corresponding vector bundle functor is $T^{*} \mathcal{B} \otimes V$, where $V$ is a vertical tangent functor. For this reason, for any fibred manifold $p: Y \rightarrow M$, the first jet prolongation $J^{1} Y \rightarrow Y$ is the affine bundle with the corresponding vector bundle $T^{*} M \otimes V Y$. Therefore, $J^{1} J^{1} Y \rightarrow J^{1} Y$ is the affine bundle with corresponding vector bundle $T^{*} M \otimes V J^{1} Y$. Thus the set of all $\mathcal{F} \mathcal{M}_{m, n}$-natural operators

$$
C: J^{1} \times Q_{\tau}(\mathcal{B}) \rightsquigarrow J^{1}\left(J^{1} \rightarrow \mathcal{B}\right)
$$

transforming admissible pairs $(\Gamma, \Lambda)$ on fibred manifolds $p: Y \rightarrow M$ into general connections $C_{Y}(\Gamma, \Lambda): J^{1} Y \rightarrow J^{1} J^{1} Y$ on $J^{1} Y \rightarrow M$ possesses the affine space structure.

Let

$$
\tilde{\Delta}:=\mathcal{J}^{1}-P: J^{1} \times Q_{\tau}(\mathcal{B}) \rightsquigarrow\left(J^{1}, T^{*} \mathcal{B} \otimes V J^{1}\right)
$$

be an $\mathcal{F M}_{m, n}$-natural operator transforming admissible pairs $(\Gamma, \Lambda)$ on $p: Y \rightarrow M$ into fibred maps $\tilde{\Delta}_{Y}(\Gamma, \Lambda): J^{1} Y \rightarrow T^{*} M \otimes V J^{1} Y$ covering the identity $i d_{J^{1} Y}: J^{1} Y \rightarrow J^{1} Y$, where $V J^{1} Y=V\left(J^{1} Y \rightarrow M\right)$ is the vertical bundle of $J^{1} Y \rightarrow M$.

By theorems presented in the monograph [4] it follows that the $\mathcal{F} \mathcal{M}_{m, n^{-}}$ natural operator $\tilde{\Delta}: J^{1} \times Q_{\tau}(\mathcal{B}) \rightsquigarrow\left(J^{1}, T^{*} \mathcal{B} \otimes V J^{1}\right)$ is of finite order.

Then the equality (1) can be written in the following form

$$
\mathcal{J}^{1}-C=t \cdot\left(\mathcal{J}^{1}-P\right) .
$$

If we denote $E:=\mathcal{J}^{1}-C$, then we can interpret the equality (2) in the following way.

Any $\mathcal{F} \mathcal{M}_{m, n}$-natural operator

$$
E: J^{1} \times Q_{\tau}(\mathcal{B}) \rightsquigarrow\left(J^{1}, T^{*} \mathcal{B} \otimes V J^{1}\right)
$$

transforming admissible pairs $(\Gamma, \Lambda)$ on $p: Y \rightarrow M$ into fibred maps $E_{Y}(\Gamma, \Lambda): J^{1} Y \rightarrow T^{*} M \otimes V J^{1} Y$ covering the identity $i d_{J^{1} Y}: J^{1} Y \rightarrow J^{1} Y$ is of the form

$$
E=t \cdot \tilde{\Delta} \text {. }
$$

Let

$$
J^{<q>}:=J^{1} \underbrace{\times_{\mathcal{M} f_{m}} \cdots \times_{\mathcal{M} f_{m}}}_{q \text {-times }} J^{1}: \mathcal{F} \mathcal{M}_{m, n} \rightarrow \mathcal{F M}
$$

be the bundle functor transforming $\mathcal{F M}_{m, n}$-objects $Y \rightarrow M$ into fibred products

$$
J^{<q>} Y:=J^{1} Y \underbrace{\times_{M} \cdots \times_{M}}_{q \text {-times }} J^{1} Y
$$


of $q$ copies of $J^{1} Y \rightarrow M$ and $\mathcal{F} \mathcal{M}_{m, n}$-maps $f: Y \rightarrow Y_{1}$ covering $f: M \rightarrow M_{1}$ into the induced fibred maps

$$
J^{<q>} f:=J^{1} f \underbrace{\times_{\underline{f}} \cdots \times_{f}}_{q \text {-times }} J^{1} f: J^{<q>} Y \rightarrow J^{<q>} Y_{1} .
$$

5. The classification of constructions of general connections on the fibred product of $\boldsymbol{q}$ copies of the first jet prolongation. We want to describe all $\mathcal{F} \mathcal{M}_{m, n}$-natural operators

$$
A: J^{1} \times Q_{\tau}(\mathcal{B}) \rightsquigarrow J^{1}\left(J^{<q>} \rightarrow \mathcal{B}\right)
$$

transforming admissible pairs $(\Gamma, \Lambda)$ on $p: Y \rightarrow M$ into general connections $A_{Y}(\Gamma, \Lambda): J^{<q>} Y \rightarrow J^{1}\left(J^{<q>} Y\right)$ on $J^{<q>} Y \rightarrow M$.

An example of such $A$ is the $\mathcal{F} \mathcal{M}_{m, n}$-natural operator $\mathcal{J}^{<q>}$ of finite order constructed by I. Kolár.

By theorems presented in the monograph [4] it follows that $\mathcal{F} \mathcal{M}_{m, n^{-}}$ natural operators $A: J^{1} \times Q_{\tau}(\mathcal{B}) \rightsquigarrow J^{1}\left(J^{<q>} \rightarrow \mathcal{B}\right)$ are of finite order.

Next, $J^{1}\left(J^{<q>} Y\right) \rightarrow J^{<q>} Y$ is the affine bundle with corresponding vector bundle $T^{*} M \otimes V J^{<q>} Y$, where $V J^{<q>} Y=V\left(J^{<q>} Y \rightarrow M\right)$ is the vertical bundle of $J^{<q>} Y \rightarrow M$. Therefore, we obtain the following $\mathcal{F} \mathcal{M}_{m, n^{-}}$ natural operator

$$
\Delta: J^{1} \times Q_{\tau}(\mathcal{B}) \rightsquigarrow\left(J^{<q>}, T^{*} \mathcal{B} \otimes V J^{<q>}\right)
$$

of finite order transforming admissible pairs $(\Gamma, \Lambda)$ on $p: Y \rightarrow M$ into fibred maps $\Delta_{Y}(\Gamma, \Lambda): J^{<q>} Y \rightarrow T^{*} M \otimes V J^{<q>} Y$ covering the identity map of $J^{<q>} Y$ given by

$$
\Delta_{Y}(\Gamma, \Lambda):=A_{Y}(\Gamma, \Lambda)-\mathcal{J}_{Y}^{<q>}(\Gamma, \Lambda) .
$$

The natural operator $A$ is completely described by the natural operator $\Delta$, because it holds

$$
A_{Y}(\Gamma, \Lambda)=\Delta_{Y}(\Gamma, \Lambda)+\mathcal{J}_{Y}^{<q>}(\Gamma, \Lambda)
$$

for any admissible pair $(\Gamma, \Lambda)$. In other words, it holds $A=\Delta+\mathcal{J}^{<q>}$.

Therefore, in order to determine all $\mathcal{F} \mathcal{M}_{m, n}$-natural operators $A: J^{1} \times Q_{\tau}(\mathcal{B}) \rightsquigarrow J^{1}\left(J^{<q>} \rightarrow \mathcal{B}\right)$ it is sufficient to describe all $\mathcal{F} \mathcal{M}_{m, n^{-}}$ natural operators $\Delta: J^{1} \times Q_{\tau}(\mathcal{B}) \rightsquigarrow\left(J^{<q>}, T^{*} \mathcal{B} \otimes V J^{<q>}\right)$.

Using the following identifications

$$
\begin{aligned}
& V J^{<q>} Y=V J^{1} Y \underbrace{\times_{M} \cdots \times_{M}}_{q \text {-times }} V J^{1} Y, \\
& T^{*} M \otimes V J^{<q>} Y=\left(T^{*} M \otimes V J^{1} Y\right) \underbrace{\times_{M} \cdots \times_{M}}_{q \text {-times }}\left(T^{*} M \otimes V J^{1} Y\right),
\end{aligned}
$$

we find out that any fibred map

$$
\tau: J^{<q>} Y \rightarrow T^{*} M \otimes V J^{<q>} Y
$$


is the system $\tau=\left(\tau_{1}, \ldots, \tau_{q}\right)$ of fibred maps

$$
\tau_{i}:=\left(i d_{T^{*} M} \otimes V p_{i}\right) \circ \tau: J^{<q>} Y \rightarrow T^{*} M \otimes V J^{1} Y
$$

covering the usual projection $p_{i}: J^{<q>} Y \rightarrow J^{1} Y$ onto an $i$-th factor of the fibred product $J^{<q>} Y=J^{1} Y \underbrace{\times_{M} \cdots \times_{M}}_{q \text {-times }} J^{1} Y, i=1, \ldots, q$.

So, the $\mathcal{F} \mathcal{M}_{m, n}$-natural operators $A: J^{1} \times Q_{\tau}(\mathcal{B}) \rightsquigarrow J^{1}\left(J^{<q>} \rightarrow \mathcal{B}\right)$ are in a bijective correspondence with systems $\left(B^{1}, \ldots, B^{q}\right)$ of $\mathcal{F} \mathcal{M}_{m, n}$-natural operators

$$
B^{i}: J^{1} \times Q_{\tau}(\mathcal{B}) \rightsquigarrow\left(J^{<q>}, T^{*} \mathcal{B} \otimes V J^{1}\right)
$$

transforming admissible pairs $(\Gamma, \Lambda)$ on $Y \rightarrow M$ into fibred maps $B_{Y}^{i}(\Gamma, \Lambda): J^{<q>} Y \rightarrow T^{*} M \otimes V J^{1} Y$ given by

$$
B_{Y}^{i}(\Gamma, \Lambda)=\left(i d_{T^{*} M} \otimes V p_{i}\right) \circ \Delta_{Y}(\Gamma, \Lambda)
$$

covering $p_{i}: J^{<q>} Y \rightarrow J^{1} Y$ for $i=1, \ldots, q$.

By theorems presented in the monograph [4] it follows that $\mathcal{F M}_{m, n^{-}}$ natural operators $B^{i}: J^{1} \times Q_{\tau}(\mathcal{B}) \rightsquigarrow\left(J^{<q>}, T^{*} \mathcal{B} \otimes V J^{1}\right)$ are of finite order.

Therefore, in order to determine all $\mathcal{F M}_{m, n}$-natural operators $A: J^{1} \times Q_{\tau}(\mathcal{B}) \rightsquigarrow J^{1}\left(J^{<q>} \rightarrow \mathcal{B}\right)$ it is sufficient to describe all $\mathcal{F} \mathcal{M}_{m, n^{-}}$ natural operators $B^{i}: J^{1} \times Q_{\tau}(\mathcal{B}) \rightsquigarrow\left(J^{<q>}, T^{*} \mathcal{B} \otimes V J^{1}\right)$ of the same type for $i=1, \ldots, q$.

Therefore, in order to determine all $\mathcal{F M}_{m, n}$-natural operators $A: J^{1} \times Q_{\tau}(\mathcal{B}) \rightsquigarrow J^{1}\left(J^{<q>} \rightarrow \mathcal{B}\right)$ it is sufficient to describe all $\mathcal{F} \mathcal{M}_{m, n^{-}}$ natural operators

$$
B: J^{1} \times Q_{\tau}(\mathcal{B}) \rightsquigarrow\left(J^{<q>}, T^{*} \mathcal{B} \otimes V J^{1}\right)
$$

transforming admissible pairs $(\Gamma, \Lambda)$ on $p: Y \rightarrow M$ into fibred maps $B_{Y}(\Gamma, \Lambda): J^{<q>} Y \rightarrow T^{*} M \otimes V J^{1} Y$ given by

$$
B_{Y}(\Gamma, \Lambda)=\left(i d_{T^{*} M} \otimes V p_{1}\right) \circ \Delta_{Y}(\Gamma, \Lambda)
$$

covering $i d_{Y}: Y \rightarrow Y$.

By theorems presented in the monograph [4] it follows that $\mathcal{F} \mathcal{M}_{m, n^{-}}$ natural operators $B: J^{1} \times Q_{\tau}(\mathcal{B}) \rightsquigarrow\left(J^{<q>}, T^{*} \mathcal{B} \otimes V J^{1}\right)$ are of finite order.

Consider the map $\delta: J^{1} Y \rightarrow J^{1} Y \underbrace{\times_{M} \cdots \times_{M}}_{q \text {-times }} J^{1} Y$ given by

$$
\delta(u)=(u, \ldots, u)
$$

for any element $u \in J_{x}^{1} Y$, where $x \in M$. Then the $\mathcal{F} \mathcal{M}_{m, n}$-natural operator $B$ defines an $\mathcal{F} \mathcal{M}_{m, n}$-natural operator

$$
B \circ \delta: J^{1} \times Q_{\tau}(\mathcal{B}) \rightsquigarrow\left(J^{1}, T^{*} \mathcal{B} \otimes V J^{1}\right)
$$

of finite order transforming admissible pairs $(\Gamma, \Lambda)$ on $p: Y \rightarrow M$ into fibred maps $(B \circ \delta)_{Y}(\Gamma, \Lambda): J^{1} Y \rightarrow T^{*} M \otimes V J^{1} Y$ given by

$$
(B \circ \delta)_{Y}(\Gamma, \Lambda):=B_{Y}(\Gamma, \Lambda) \circ \delta
$$


covering the identity $i d_{J^{1} Y}$.

Hence we see that $B \circ \delta=t \cdot \tilde{\Delta}$ for the real number $t$, i.e.

$$
B_{Y}(\Gamma, \Lambda)(u, \ldots, u)=t \cdot \tilde{\Delta}_{Y}(\Gamma, \Lambda)(u)
$$

for any admissible pair $(\Gamma, \Lambda)$ on $Y \rightarrow M$ and for any element $u \in J_{x}^{1} Y$, where $x \in M$.

We have the projection $V \pi_{0}^{1}: V J^{1} Y \rightarrow V Y$, where $\pi_{0}^{1}: J^{1} Y \rightarrow Y$ is the jet projection. Then the $\mathcal{F} \mathcal{M}_{m, n}$-natural operator $B$ defines an $\mathcal{F} \mathcal{M}_{m, n^{-}}$ natural operator

$$
D:=\left(i d_{T^{*} M} \otimes V \pi_{0}^{1}\right) \circ B: J^{1} \times Q_{\tau}(\mathcal{B}) \rightsquigarrow\left(J^{<q>}, T^{*} \mathcal{B} \otimes V\right)
$$

transforming admissible pairs $(\Gamma, \Lambda)$ on $p: Y \rightarrow M$ into fibred maps $D_{Y}(\Gamma, \Lambda): J^{<q>} Y \rightarrow T^{*} M \otimes V Y$ given by

$$
D_{Y}(\Gamma, \Lambda)=\left(i d_{T^{*} M} \otimes V \pi_{0}^{1}\right) \circ B_{Y}(\Gamma, \Lambda)
$$

covering the projection $\pi_{0}^{1} \circ p_{1}$.

By theorems presented in the monograph [4] it follows that the $\mathcal{F} \mathcal{M}_{m, n}$-natural operator $D: J^{1} \times Q_{\tau}(\mathcal{B}) \rightsquigarrow\left(J^{<q>}, T^{*} \mathcal{B} \otimes V\right)$ is of finite order.

Because of the invariance of $D$ with respect to fibred manifold charts, the existence of $\left(\Gamma, \Lambda, y_{0}, r\right)$-quasi-normal fibred coordinate systems and the non-linear Peetre theorem (see [4]), we deduce that $D$ is determined by the values

$$
\begin{aligned}
D_{Y}\left(\Gamma_{0}+\sum_{k=1}^{n} \sum_{j=1}^{m} \sum_{|\alpha|+|\beta| \leq r-1} \Gamma_{j \alpha \beta}^{k} x^{\alpha} y^{\beta} d x^{j} \otimes \frac{\partial}{\partial y^{k}},\right. \\
\left.\left(\sum_{1 \leq|\gamma| \leq s} \Lambda_{i_{2} i_{3} \gamma}^{i_{1}} x^{\gamma}\right)_{i_{2}, i_{3}=1, \ldots, m}^{i_{1}=1, \ldots, m}\right)(\tilde{u})
\end{aligned}
$$

from $T_{0}^{*} \mathbb{R}^{m} \otimes V_{(0,0)} \mathbb{R}^{m, n}$ for all $\tilde{u}=\left(u_{1}, \ldots, u_{q}\right)$ such that $u_{1}, \ldots, u_{q} \in$ $\left(J^{1} \mathbb{R}^{m, n}\right)_{(0,0)}$, all natural numbers $r, s=1,2, \ldots$, all $\Lambda_{i_{2} i_{3} \gamma}^{i_{1}} \in \mathbb{R}$ and all $\Gamma_{j \alpha \beta}^{k} \in \mathbb{R}$ satisfying the condition

$$
\Phi_{r}\left(j_{0}^{r-1}\left(\sum_{|\alpha| \leq r-1} \sum_{j=1}^{m} \sum_{k=1}^{n} \Gamma_{j \alpha \beta}^{k} x^{\alpha} d x^{j} \otimes e_{k}\right)\right)=0
$$

for any multiindex $\beta \in \mathbb{N}^{n}$ such that $|\beta| \leq r-1$, where $\Gamma_{0}=\sum_{i=1}^{m} d x^{i} \otimes \frac{\partial}{\partial x^{i}}$ is a trivial general connection on the fibred manifold $\mathbb{R}^{m, n}$.

Using the invariance of $D$ with respect to the homotheties $t \cdot i d_{\mathbb{R}^{m, n}}$ for $t>0$ (they preserve $u_{1}, \ldots, u_{q}$ ) and next applying the homogeneous function theorem (we can apply it because of the condition (4)) and putting $t \rightarrow 0$, we see that every value (3) is equal to

$$
D_{Y}\left(\Gamma_{0}, \Lambda^{0}\right)\left(u_{1}, \ldots, u_{q}\right) \in T_{0}^{*} \mathbb{R}^{m} \otimes V_{(0,0)} \mathbb{R}^{m, n},
$$


where $\Lambda^{0}$ is a flat torsion-free classical linear connection on $\mathbb{R}^{m}$.

Consider a tangent vector $\xi \in T_{0} \mathbb{R}^{m}$ and elements $u_{1}=j_{0}^{1}\left(i d_{\mathbb{R}^{m}}, \rho\right) \in$ $\left(J^{1} \mathbb{R}^{m, n}\right)_{(0,0)}, u_{2}, \ldots, u_{q} \in\left(J^{1} \mathbb{R}^{m, n}\right)_{(0,0)}$ for a map $\rho=\left(\rho_{1}, \ldots, \rho_{n}\right): \mathbb{R}^{m} \rightarrow$ $\mathbb{R}^{n}$ such that $j_{0}^{1}\left(\rho_{a}\right) \neq 0$ for $a=1, \ldots, n$.

Write

$$
B_{Y}\left(\Gamma_{0}, \Lambda^{0}\right)\left(u_{1}, \ldots, u_{q}\right)(\xi):=\left.\frac{d}{d t}\right|_{t=0}\left(j_{0}^{1}\left(i d_{\mathbb{R}^{m}}, \rho+t v\right)\right)
$$

for some function $v=\left(v_{1}, \ldots, v_{n}\right): \mathbb{R}^{m} \rightarrow \mathbb{R}^{n}$ and

$$
v_{a}(0):=v_{a}^{0}
$$

for $a=1, \ldots, n$. Then

$$
D_{Y}\left(\Gamma_{0}, \Lambda^{0}\right)\left(u_{1}, \ldots, u_{q}\right)(\xi)=\left.\frac{d}{d t}\right|_{t=0}\left(0, t v_{a}^{0}\right) .
$$

The fibred map

$$
\bar{x}^{i}=x^{i}, \bar{y}^{k}=y^{k}+\left(y^{k}\right)^{2}
$$

preserves: the trivial general connection $\Gamma_{0}$, the flat torsion-free classical linear connection $\Lambda^{0}$, the $\mathcal{F} \mathcal{M}_{m, n}$-natural operator $B$, elements $u_{1}, \ldots, u_{q}$, the vector $\xi$ and sends $\left.\frac{d}{d t}\right|_{t=0}\left(j_{0}^{1}\left(i d_{\mathbb{R}^{m}}, \rho+t v\right)\right)$ into $\left.\frac{d}{d t}\right|_{t=0}\left(j_{0}^{1}\left(\rho+\rho_{a}^{2}+2 t\left(\frac{1}{2} v+\right.\right.\right.$ $\left.\left.\left.\rho_{a} v_{a}^{0}+\frac{1}{2} t\left(v_{a}^{0}\right)^{2}\right)\right)\right)$. Then it holds $v_{a}^{0}=0$ for $a=1, \ldots, n$. Hence we have the equality

$$
D_{Y}\left(\Gamma_{0}, \Lambda^{0}\right)\left(u_{1}, \ldots, u_{q}\right)(\xi)=0
$$

Consequently,

$$
D: J^{1} \times Q_{\tau}(\mathcal{B}) \rightsquigarrow\left(J^{<q>}, T^{*} \mathcal{B} \otimes V\right)
$$

is the zero operator.

Consider the well-known exact sequence

$$
0 \rightarrow T^{*} M \otimes V Y \rightarrow V J^{1} Y \rightarrow V Y \rightarrow 0
$$

over $J^{1} Y$. Next we obtain the following exact sequence

$$
0 \rightarrow T^{*} M \otimes T^{*} M \otimes V Y \rightarrow T^{*} M \otimes V J^{1} Y \rightarrow T^{*} M \otimes V Y \rightarrow 0
$$

over $J^{1} Y$.

Therefore, the $\mathcal{F} \mathcal{M}_{m, n}$-natural operator $B$ can be interpreted as an $\mathcal{F} \mathcal{M}_{m, n}$-natural operator

$$
B: J^{1} \times Q_{\tau}(\mathcal{B}) \rightsquigarrow\left(J^{<q>}, T^{*} \mathcal{B} \otimes T^{*} \mathcal{B} \otimes V\right)
$$

of finite order transforming admissible pairs $(\Gamma, \Lambda)$ on $p: Y \rightarrow M$ into fibred maps $B_{Y}(\Gamma, \Lambda): J^{<q>} Y \rightarrow T^{*} M \otimes T^{*} M \otimes V Y$ covering the projection $\pi_{0}^{1} \circ p_{1}$. 
Using the invariance of the $\mathcal{F} \mathcal{M}_{m, n}$-natural operator $B$ with respect to fibred manifold charts, the existence of $\left(\Gamma, \Lambda, y_{0}, r\right)$-quasi-normal fibred coordinate systems and non-linear Peetre theorem, we deduce that the $\mathcal{F} \mathcal{M}_{m, n^{-}}$ natural operator $B$ is determined by the values

$$
B_{Y}\left(\Gamma_{0}+\sum_{k=1}^{n} \sum_{j=1}^{m} \sum_{|\alpha|+|\beta| \leq r-1} \Gamma_{j \alpha \beta}^{k} x^{\alpha} y^{\beta} d x^{j} \otimes \frac{\partial}{\partial y^{k}},\right.
$$

$$
\left.\left(\sum_{1 \leq|\gamma| \leq s} \Lambda_{i_{2} i_{3} \gamma}^{i_{1}} x^{\gamma}\right)_{i_{2}, i_{3}=1, \ldots, m}^{i_{1}=1, \ldots, m}\right)(\tilde{u})
$$

from $T_{0}^{*} \mathbb{R}^{m} \otimes T_{0}^{*} \mathbb{R}^{m} \otimes V_{(0,0)} \mathbb{R}^{m, n}$ for all elements $\tilde{u}=\left(u_{1}, \ldots, u_{q}\right)$ such that $u_{1}, \ldots, u_{q} \in\left(J^{1} \mathbb{R}^{m, n}\right)_{(0,0)}$, all natural numbers $r, s=1,2, \ldots$, all numbers $\Lambda_{i_{2} i_{3} \gamma}^{i_{1}} \in \mathbb{R}$ and all numbers $\Gamma_{j \alpha \beta}^{k} \in \mathbb{R}$ satisfying the condition (4) for any multiindex $\beta \in \mathbb{N}^{n}$ such that $|\beta| \leq r-1$.

We use the following identifications

$$
\begin{aligned}
& \left(J^{1} \mathbb{R}^{m, n}\right)_{(0,0)} \cong \mathbb{R}^{m *} \otimes \mathbb{R}^{n}, \\
& T_{0}^{*} \mathbb{R}^{m} \otimes T_{0}^{*} \mathbb{R}^{m} \otimes V_{(0,0)} \mathbb{R}^{m, n} \cong \mathbb{R}^{m *} \otimes \mathbb{R}^{m *} \otimes \mathbb{R}^{n} .
\end{aligned}
$$

Using the invariance of the $\mathcal{F} \mathcal{M}_{m, n}$-natural operator $B$ with respect to the homotheties $t \cdot i d_{\mathbb{R}^{m, n}}$ for $t>0$ (they preserve the elements $u_{1}, \ldots, u_{q}$ ) and next applying the homogeneous function theorem (we can apply it because of the condition (4)), we observe that every value (6) is equal to

$$
\begin{aligned}
B_{Y}\left(\Gamma_{0}\right. & +\sum_{k, l=1}^{n} \sum_{j=1}^{m} \Gamma_{j l}^{k} y^{l} d x^{j} \otimes \frac{\partial}{\partial y^{k}} \\
& \left.+\sum_{k=1}^{n} \sum_{i, j=1}^{m} \Gamma_{j i}^{k} x^{i} d x^{j} \otimes \frac{\partial}{\partial y^{k}}, \Lambda^{0}\right)\left(u_{1}, \ldots, u_{q}\right),
\end{aligned}
$$

where $\Gamma_{j l}^{k}:=\Gamma_{j(0) e_{l}}^{k} \in \mathbb{R}$ and $\Gamma_{j i}^{k}:=\Gamma_{j e_{i}(0)}^{k} \in \mathbb{R}$. Of course, $\Gamma_{j i}^{k}=-\Gamma_{i j}^{k}$. Moreover, the value (7) is linear in $\Gamma_{j i}^{k}$ and $\Gamma_{j l}^{k}$ with coefficients being smooth functions in $\left(u_{1}, \ldots, u_{q}\right)$.

In particular, it holds

$$
\begin{array}{r}
B_{Y}\left(\Gamma_{0}+y^{l} d x^{j} \otimes \frac{\partial}{\partial y^{k}}, \Lambda^{0}\right)\left(u_{1}, \ldots, u_{q}\right) \\
\quad=B_{Y}\left(\Gamma_{0}+d x^{j} \otimes \tilde{Y}, \Lambda^{0}\right)\left(u_{1}, \ldots, u_{q}\right)
\end{array}
$$

for $\tilde{Y}=\left(y^{l}+1\right) \frac{\partial}{\partial y^{k}}$. Since $Y_{0}:=\frac{\partial}{\partial y^{k}}|0| 0$, there is a local diffeomorphism $H: \mathbb{R}^{n} \rightarrow \mathbb{R}^{n}$ such that an element $j_{0}^{1} H=i d$ and $H_{*} \tilde{Y}=\frac{\partial}{\partial y^{k}}$ near 0 .

The map $i d_{\mathbb{R}^{m}} \times H$ preserves elements $u_{1}, \ldots, u_{q}$, the $\mathcal{F} \mathcal{M}_{m, n}$-natural operator $B$ and the connection $\Lambda^{0}$, and sends $\Gamma_{0}+d x^{j} \otimes \tilde{Y}$ into $\Gamma_{0}+d x^{j} \otimes \frac{\partial}{\partial y^{k}}$ and acts on $T_{0}^{*} \mathbb{R}^{m} \otimes T_{0}^{*} \mathbb{R}^{m} \otimes V_{(0,0)} \mathbb{R}^{m, n}$ as the identity map. Then using 
the invariance of the $\mathcal{F} \mathcal{M}_{m, n}$-natural operator $B$ with respect to $i d_{\mathbb{R}^{m}} \times H$, we see that

$$
B_{Y}\left(\Gamma_{0}+d x^{j} \otimes \tilde{Y}, \Lambda^{0}\right)\left(u_{1}, \ldots, u_{q}\right)=B_{Y}\left(\Gamma_{0}+d x^{j} \otimes \frac{\partial}{\partial y^{k}}, \Lambda^{0}\right)\left(u_{1}, \ldots, u_{q}\right) .
$$

From (8) we obtain

$$
B_{Y}\left(\Gamma_{0}+d x^{j} \otimes \frac{\partial}{\partial y^{k}}, \Lambda^{0}\right)\left(u_{1}, \ldots, u_{q}\right)=B_{Y}\left(\Gamma_{0}, \Lambda^{0}\right)\left(u_{1}, \ldots, u_{q}\right)=0 .
$$

Therefore, it holds

$$
B_{Y}\left(\Gamma_{0}+y^{l} d x^{j} \otimes \frac{\partial}{\partial y^{k}}, \Lambda^{0}\right)\left(u_{1}, \ldots, u_{q}\right)=0 .
$$

Consequently, the values (6) are equal to

$$
\sum f_{k}^{j i}\left(u_{1}, \ldots, u_{q}\right) \Gamma_{j i}^{k}
$$

for some smooth functions $f_{k}^{j i}$.

Using the invariance of $\mathcal{F} \mathcal{M}_{m, n}$-natural operator $B$ with respect to fibre homotheties $i d_{\mathbb{R}^{m}} \times t \cdot i d_{\mathbb{R}^{n}}$ for $t>0$, we get the homogeneous conditions

$$
t \cdot f_{k}^{j i}\left(t u_{1}, \ldots, t u_{q}\right)=t \cdot f_{k}^{j i}\left(u_{1}, \ldots, u_{q}\right) .
$$

Cancelling both sides by $t$ and putting $t \rightarrow 0$, we see that functions $f_{k}^{j i}$ are constants.

Thus the $\mathcal{F} \mathcal{M}_{m, n}$-natural operator $B$ is determined by the values

$$
B_{Y}\left(\Gamma_{0}+x^{i} d x^{j} \otimes \frac{\partial}{\partial y^{k}}-x^{j} d x^{i} \otimes \frac{\partial}{\partial y^{k}}, \Lambda^{0}\right)(0, \ldots, 0)
$$

for $1 \leq i<j \leq m$ and $k=1, \ldots, n$. In other words, we claim that the $\mathcal{F} \mathcal{M}_{m, n}$-natural operator $B$ is determined by the $\mathcal{F} \mathcal{M}_{m, n}$-natural operator $B \circ \delta$, where the map $\delta: J^{1} Y \rightarrow J^{<q>} Y$ is given by $\delta(u)=(u, \ldots, u)$. As we observed earlier, the equality $B \circ \delta=t \cdot \tilde{\Delta}$ holds for some $t \in \mathbb{R}$. It means that $\mathcal{F} \mathcal{M}_{m, n}$-natural operators $B \circ \delta$ form 1-parameter family of operators. Of course, any $\tilde{\Delta} \circ p_{i}$ is an example of a such $B$ for $i=1, \ldots, q$. In particular, $B$ is proportional to $\tilde{\Delta} \circ p_{1}$ and similarly $B^{i}$ is proportional to $\tilde{\Delta} \circ p_{i}$ for $i=1, \ldots, q$. Thus we proved the following classification theorem.

Theorem 2. The $\mathcal{F} \mathcal{M}_{m, n}$-natural operators

$$
A: J^{1} \times Q_{\tau}(\mathcal{B}) \rightsquigarrow J^{1}\left(J^{<q>} \rightarrow \mathcal{B}\right)
$$

transforming admissible pairs $(\Gamma, \Lambda)$ on $\mathcal{F M}_{m, n}$-objects $p: Y \rightarrow M$ into general connections $A_{Y}(\Gamma, \Lambda): J^{<q>} Y \rightarrow J^{1}\left(J^{<q>} Y\right)$ on $J^{<q>} Y \rightarrow M$ form the q-parameter family

$$
\mathcal{J}^{<q>}+\left(t_{i} \cdot \tilde{\Delta} \circ p_{i}\right)_{i=1, \ldots, q}
$$

for real numbers $t_{i}$. 
Remark 1. The $q$-parameter family (9) can be written equivalently in the following form:

$$
\left(\mathcal{J}^{<q>}+t_{1} \cdot \tilde{\Delta}, \mathcal{J}^{<q>}+t_{2} \cdot \tilde{\Delta}, \ldots, \mathcal{J}^{<q>}+t_{q} \cdot \tilde{\Delta}\right) .
$$

The curvature

$$
\mathcal{R}_{Y}(\Gamma): Y \rightarrow \bigwedge^{2} T^{*} M \otimes V Y
$$

can be treated as the fibred map

$$
\mathcal{R}_{Y}(\Gamma): J^{1} Y \rightarrow T^{*} M \otimes T^{*} M \otimes V Y .
$$

Moreover, by the exact sequence (5) the curvature can be treated as the fibred map

$$
\mathcal{R}_{Y}(\Gamma): J^{1} Y \rightarrow T^{*} M \otimes V J^{1} Y .
$$

Thus we obtain an $\mathcal{F} \mathcal{M}_{m, n}$-natural operator

$$
\mathcal{R}: J^{1} \times Q_{\tau}(\mathcal{B}) \rightsquigarrow\left(J^{1}, T^{*} \mathcal{B} \otimes V J^{1}\right) .
$$

By theorems presented in the monograph [4] it follows that the $\mathcal{F} \mathcal{M}_{m, n}$-natural operator $\mathcal{R}: J^{1} \times Q_{\tau}(\mathcal{B}) \rightsquigarrow\left(J^{1}, T^{*} \mathcal{B} \otimes V J^{1}\right)$ is of finite order.

Clearly, we can use $\mathcal{R}$ instead of $\tilde{\Delta}$ in Theorem 2. Because of Theorem 2 for $q=1$ we conclude that $\mathcal{R}$ is proportional to $\tilde{\Delta}$. Therefore, we can reformulate Theorem 2 in the following way.

Theorem 3. The $\mathcal{F} \mathcal{M}_{m, n}$-natural operators

$$
A: J^{1} \times Q_{\tau}(\mathcal{B}) \rightsquigarrow J^{1}\left(J^{<q>} \rightarrow \mathcal{B}\right)
$$

transforming admissible pairs $(\Gamma, \Lambda)$ on $\mathcal{F M}_{m, n}$-objects $p: Y \rightarrow M$ into general connections $A_{Y}(\Gamma, \Lambda): J^{<q>} Y \rightarrow J^{1}\left(J^{<q>} Y\right)$ on $J^{<q>} Y \rightarrow M$ form the q-parameter family

$$
\mathcal{J}^{<q>}+\left(t_{i} \cdot \mathcal{R} \circ p_{i}\right)_{i=1, \ldots, q}
$$

for real numbers $t_{i}$, where $\mathcal{R}$ is the $\mathcal{F} \mathcal{M}_{m, n}$-natural operator from (11).

\section{REFERENCES}

[1] Doupovec, M., Mikulski, W. M., Holonomic extension of connections and symmetrization of jets, Rep. Math. Phys. 60 (2007), 299-316.

[2] Kolár, I., Prolongations of generalized connections, in: Differential Geometry (Budapest, 1979), Colloq. Math. Soc. János Bolyai, 31, North-Holland, Amsterdam, 1982, 317-325.

[3] Kolár̆, I., Higher order absolute differentiation with respect to generalized connections, in: Differential Geometry (Warsaw, 1979), PWN - Polish Sci. Publ., Warszawa, 1984, $153-162$.

[4] Kolář, I., Michor, P. W., Slovák J., Natural Operations in Differential Geometry, Springer-Verlag, Berlin, 1993.

[5] Kurek, J., Mikulski, W. M., On prolongations of projectable connections, Ann. Polon. Math. 101 (3) (2011), 237-250. 
[6] Mikulski, W. M., On "special" fibred coordinates for general and classical connections, Ann. Polon. Math. 99 (2010), 99-105.

[7] Mikulski, W. M., On prolongation of connections, Ann. Polon. Math. 97 (2) (2010), 101-121.

[8] Plaszczyk, M., The constructions of general connections on second jet prolongation, Ann. Univ. Mariae Curie-Skłodowska Sect. A 68 (1) (2014), 67-89.

Mariusz Plaszczyk

Institute of Mathematics

Maria Curie-Skłodowska University

pl. M. Curie-Skłodowskiej 1

20-031 Lublin

Poland

e-mail: mariusz.plaszczyk@poczta.umcs.lublin.pl

Received April 25, 2018 